\title{
Circuit
}

Musiques contemporaines

\section{Anges noirs, spectacle du Quatuor Bozzini}

\section{Richard Simas}

Volume 22, numéro 1, 2012

URI : https://id.erudit.org/iderudit/1008971ar

DOI : https://doi.org/10.7202/1008971ar

Aller au sommaire du numéro

Éditeur(s)

Les Presses de l’Université de Montréal

ISSN

1183-1693 (imprimé)

1488-9692 (numérique)

Découvrir la revue

Citer ce compte rendu

Simas, R. (2012). Compte rendu de [Anges noirs, spectacle du Quatuor Bozzini]. Circuit, 22(1), 91-96. https://doi.org/10.7202/1008971ar d'utilisation que vous pouvez consulter en ligne.

https://apropos.erudit.org/fr/usagers/politique-dutilisation/ 


\section{Anges noirs, spectacle du Quatuor Bozzini}

Richard Simas

Les pouvoirs authentiquement magiques et spirituels de la musique émanent des profondeurs de notre psyché. - George Crumb ${ }^{1}$

Dans un fondu de lumière bleue provenant des coulisses, les quatre musiciens du quatuor à cordes Bozzini apparaissent peu à peu sur scène, accoutrés en morts-vivants: visages blanchis, pieds nus, vêtements chiffonnés, chevelure hirsute, la peau constellée de plaies, brûlures et autres saletés. Les pupilles de leurs yeux sombres, vides, sont d'un blanc vitreux - comme si leurs globes oculaires s'étaient retournés vers l'intérieur. D'un clic, un soldat allume une lampe suspendue au-dessus de leurs têtes et raconte un rêve récurrent qui parle d'avions de guerre bourdonnant autour de sa tête comme de menaçants insectes. Dans le registre aigu, le quatuor joue alors les plaintes et hurlements qui amorcent Threnody I: Night of the Electric Insects - l'un des mouvements de Black Angels (Images 1): Thirteen images from the dark land (1970) de George Crumb.

Cette théâtralité faite d'images obsessives pourrait laisser perplexe, mais le Quatuor Bozzini est comme un poisson dans l'eau dans cette version hautement dramatique de la célèbre œuvre pour quatuor à cordes amplifié. Ange noir/Black Angel résulte d'une audacieuse collaboration entre le Quatuor Bozzini et le dramaturge montréalais Jean-Frédéric Messier, qui met en scène ce spectacle en plus de signer le texte - lequel colle intimement à la partition de Black Angels.

Il s'agit sans doute du seul quatuor à cordes pour lequel le compositeur s'est inspiré de la guerre du Vietnam². L'œuvre s'abreuve à une panoplie de sources métaphysiques de même qu'au symbolisme numérologique afin de créer une obsédante "parabole de notre monde contemporain troublé3». Black Angels compte trois mouvements qui décrivent un «voyage de l'âme »:
1. George Crumb (1980), "Music: Does it have a future?", The Kenyon Review.

2. [ndlr] Partition éditée par C. F. Peters (P66304); parmi une douzaine d'enregistrements disponibles, on citera le plus récent, interprété par le Quatuor Miro (Bridge Records 9139, 2003).

3. Black Angels, notes de programme, livret du CD Elektra Nonesuch 755979242, 1990 
Départ (chute en disgrâce), Absence (annihilation spirituelle), et Retour (rédemption). Sa structure est «une immense forme en arche suspendue aux trois "Threnodies"4».

Il y a quarante ans, amplifier électriquement les instruments à cordes était un geste provocant, conçu pour insuffler à l'œuvre un caractère hautement surréaliste. L'œuvre originale de Crumb fait aussi crier, chanter, siffler, soupirer les musiciens qui jouent aussi des gongs, maracas et verres de cristal, ce qui crée un effet puissamment dramatique.

Ce projet a commencé en 2007, quand le Quatuor Bozzini a commandé à $\mathrm{M}$. Messier un texte qui fut ensuite traduit en allemand pour être lu dans le cadre d'un concert, à l'occasion d'un festival en Autriche. Quatre ans plus tard, le théâtre Momentum et le Quatuor Bozzini créaient l'intégrale d’Ange noir/Black Angel (interprétée dans ses deux versions, anglaise et française) du $1^{\text {er }}$ au 3 juin 2011 au Théâtre de Quat'Sous (Montréal), lors de la cinquième édition de l'OFFTA - Festival d'arts vivants.

Le résultat: une pièce de théâtre où se glisse un concert de musique contemporaine. Bien que le monologue du soldat, créé de toutes pièces par Messier, ne réponde à aucune indication contenue dans la partition de Crumb, le périple du personnage suit un itinéraire tout à fait parallèle à celui que décrit le texte musical. Le spectacle dure environ cinquante-quatre minutes - le double de la version concert. Si chacun des treize mouvements de Black Angels est joué au moins une fois sans texte parlé, certains d'entre eux sont repris et prolongés pour accompagner diverses scènes. Globalement, les choix scéniques de Messier talonnent de près les sources et les structures musicales de Crumb, qu'il souligne d'éléments visuels et dramatiques.

Sur scène, le quatuor émerge d'un «endroit où les choses sont sens dessus dessous. Il y avait de terribles choses dans l'air ${ }^{5} . . . »$, écrit Crumb en référence à ce qui, à la fin des années 1960, l'a inspiré pour cette œuvre. Sous une lumière crue, dont le concepteur d'éclairages Michel Beaulieu a su conserver la simplicité évocatrice, les musiciens créent de saisissantes images. Mais l'aventure du Quatuor Bozzini dans cette audacieuse création théâtralomusicale est typique de son habituelle hardiesse dans l'engagement quand il s'agit d'explorer l'infini cosmos du quatuor à cordes contemporain.

Lorsqu'on lui demande ce qui a motivé la création du groupe, Isabelle Bozzini a cette réponse toute simple: «Nous voulions jouer la musique de notre temps. Et il s'agissait plutôt d'une posture politique que d'une affirmation esthétique. » Fondé en 1999 et d'abord baptisé Euterpe (un nom 
que les membres fondateurs avaient adopté à l'époque de leurs études), le quatuor Bozzini a systématiquement mis au programme de ses concerts une proportion égale de musique classique et de musique contemporaine, et commandé des œuvres à de jeunes compositeurs, tels Michael Oesterle et JeanFrançois Laporte. L'appétit de ces artistes pour le risque est une constante, et cette ouverture à l'expérimentation a porté ses fruits; et c'est cela qui les distingue aujourd'hui, ici comme à l'étranger. C'est bien davantage le résultat d'un «processus organique » que celui d'une stratégie délibérée, estime la violoncelliste: «Je crois toujours que je ne fais que ce que je crois juste de faire, sans égard aux questions de goût.»

"Qu'il s'agisse de questions artistiques ou organisationnelles, notre fonctionnement demeure fondamentalement démocratique», ajoute Clemens Merkel. «Notre travail musical part lui aussi de la base: les éléments du rythme et de la basse, au violoncelle et à l'alto, sont la colonne vertébrale de notre approche musicale, au contraire de cette "domination mélodique" des registres aigus qui est bien plus courante. Les premier et second violons s'échangent constamment les rôles. En tant qu'ensemble, nous participons tous à la discussion au sujet de l'œuvre et de l'interprétation à lui donner. Ce qui nous distingue à plusieurs égards des autres quatuors à cordes et influence significativement notre son. » En plus de ses nombreux contacts et de son parcours résolument européen, Merkel a également apporté avec lui cette approche démocratique (sous l'influence d'expériences acquises en Allemagne) lorsqu'il s'est joint à l'ensemble en 1999.

Les douze ans d'histoire du quatuor ont été marqués par une série de projets singuliers, créés sur mesure pour répondre aux besoins et aux envies du groupe en matière d'expérimentation. Ange Noir/Black Angel est le plus récent élément d'une liste qui compte une collaboration du Quatuor Bozzini avec l'International Wandelweiser Group, divers projets associant musique et vidéo, le Composer's Kitchen annuel, ainsi que la fondation de CQB, sa propre maison de disques.

Fondé dans le contexte dynamique et fluide du Montréal des années 1990, le quatuor reconnaît que cette ville constitue un excellent foyer pour la musique nouvelle et expérimentale. «Je ne crois pas qu'il y ait une autre place au monde qui rivalise avec Montréal quant à son dynamisme et son ouverture à l'égard de la création contemporaine, ajoute Clemens Merkel. Ici, notre travail est reconnu, et le système de financement exige que vous créiez votre propre série de concerts, ce qui signifie que vous avez un pouvoir décisionnel total quant à votre programmation, ce qui n'est pas le cas en Europe.» 
6. [ndlr] Un autre exemple récent d'un quatuor à cordes qui inspire une production théâtrale se trouve dans le spectacle Eraritjaritjaka de Heiner Goebbels, interprété par André Wilms et le Quatuor Mondrian. Une représentation a eu lieu à Montréal dans le cadre du Festival de théâtre des Amériques, du 17 au 19 mai 2006. Voir le compte-rendu de Georges Leroux dans Spirale, no 211 (2006), p. 8-9. www. erudit.org/culture/spirale1048177/ spirale1059186/16614ac.pdf
La réputation croissante du quatuor (et la demande qui suit en proportion) permet au Quatuor Bozzini d'aller régulièrement en tournée en Europe - et plus récemment en Argentine. D'autres événements significatifs ont jalonné le parcours du groupe. Retenons surtout l'arrivée de la violoniste Nadia Francavilla en 2002, les deux saisons du Bozzini en résidence au Théâtre La Chapelle (2003-2005), et une production «pleins feux» de la SMCQ en 2006 (La Quadrature du cercle) où les membres du quatuor ont eu le sentiment de jouer dans un véritable espace public, d'intéresser l'oreille d'un auditoire élargi... et de critiques renommés.

Dans la seconde partie d'Ange Noir/Black Angel, intitulée Absence, les quatre musiciens sont assis par terre autour de Marcel Pomerleau, tandis qu'un unique tube fluorescent descend vers l'acteur, menaçant de l'écraser. Les musiciens tiennent leurs instruments et écoutent le monologue comme les témoins d'un rite sacré. Leur présence immobile revêt un poids dramatique, et avant même que le quatuor attaque le mouvement suivant, Sarabanda de la Muerte Oscura, ils sont déjà en synergie avec les images et les mots présentés sur scène. C'est là un exemple de la finesse de la direction de Messier, dans laquelle la superposition de multiples éléments narratifs (lumière, image, son, texte, partition, jeu théâtral) crée un objet d'art saisissant, qui va bien au delà des représentations théâtrales et musicales typiques.

Il est inusité qu'une composition musicale inspire un texte de théâtre. Traditionnellement, on compose plutôt la musique pour accompagner un récit préexistant. «Le texte de Jean-Fred va comme un gant à cette musique, juge Clemens Merkel. Nous sommes vraiment chanceux de collaborer avec un auteur qui lit et joue de la musique. Car il comprend ce langage et prend grand soin d'accorder l'espace nécessaire à chacun des éléments du spectacle. » C'est d'une main précise que Messier fait coexister les deux textes, musical et dramatique; c'est dans un vaste espace que peuvent se déployer l'histoire du soldat et la musique par laquelle Crumb exprime son réquisitoire métaphysique contre la guerre ${ }^{6}$.

La capacité de jouer toute la musique par cœur était un préalable essentiel pour la mise en scène d'un tel spectacle. De plus, les musiciens devaient s'adapter à des exigences et à des détails du travail théâtral qui leur étaient jusque-là étrangers. Par exemple, les lentilles de contact qui leur conféraient ces yeux fantomatiques servaient également à masquer leur regard de musiciens: on ne devait pas les voir se consulter visuellement au cours de leur prestation. «Nous avons fait confiance à Jean-Frédéric. C'était bon pour nous de lâcher prise et de tomber dans le filet de sécurité d'un autre, illustre Clemens Merkel. Cela a amené la musique dans une nouvelle dimension. » 
Comme pour répondre à la question que pose George Crumb dans son essai de 1980 ( «La musique a-t-elle un avenir?»), la position d'Isabelle Bozzini à l'égard de l'interprétation est la clé de l'expérimentation du Quatuor Bozzini : «Notre préoccupation n’est pas: “De quoi avons-nous l'air sur scène en tant qu'artistes?", mais plutôt: "Comment servons-nous la musique et le son?” Cela nous amène à approfondir notre compréhension de la musique, non à rivaliser de vélocité pour briller de mille feux... Nous nous intéressons à une musique qui favorise la réflexion et qui vient nous toucher en profondeur. L'avenir de la musique est dans sa sonorité propre, pas dans la nostalgie d'une virtuosité paganinienne. »

Cette version théâtrale de Black Angels était le défi idéal. Clemens Merkel se réjouit: «Nous avons dû marcher sur scène avec nos instruments dans le noir total. À d'autres moments, nous étions éblouis par les éclairages et nous ne pouvions pas nous apercevoir l'un l'autre comme d'habitude. Mais maintenant, nous pouvons jouer cette œuvre dans n'importe quel contexte. »

Le risque crée une dynamique essentielle pour coller à la vision du Quatuor Bozzini. "Sur scène, nous ne craignons pas de nous "planter". Un bon exemple, c'est quand nous devions jouer ce passage où Crumb cite le $14^{\mathrm{e}}$ quatuor en ré mineur de Schubert (La jeune fille et la mort). Il faut jouer avec l'instrument placé la tête en bas. L'intention est claire : ça sonnera faux. Il est pratiquement impossible de rendre une note juste avec cette contrainte. Cela exige donc que le musicien accepte de se montrer vulnérable... Eh bien! nous l'avons fait. »

À l'égard de cette notion de vulnérabilité dans le travail, les membres du Quatuor Bozzini sont implacables, et c'est peut-être ce qui, si l'on se penche sur leur histoire, les caractérise le plus fidèlement en tant qu'artistes contemporains. Dans la scène finale «Rédemption », le soldat-ange déchu a cheminé vers un monde immatériel, dans une sorte de régression psychanalytique. Le voilà nu au centre de la scène, encadré par les musiciens, éclairé à contre-jour par une lumière éthérée. C'est un moment de transformation, un voyage éternel dans le cycle de la vie et de la mort. Tandis que l'acteur se love en position fœtale, les musiciens assistent en silence au rite de passage, l'instrument dans une main, comme s'ils méditaient sur le sens de cet instant dramatique. La coda musicale d'Ange Noir/Black Angel sera faite de mots.

Je n'irai pas plus loin, mon chemin s'arrête ici. Tout est très silencieux maintenant. C'est comme si j'étais de retour dans le ventre de ma mère. Mais je n'entends pas son coeur battre ${ }^{7}$.
7. Jean-Frédéric Messier, Ange Noir/ Black Angel, 2007 
Tout comme la version concert originale d'Ange Noir/Black Angel, cette collaboration Bozzini-Momentum, bien que très différente dans sa proposition artistique, se penche fondamentalement sur l'essence profonde du bien et du mal. En présentant sur scène un acteur nu et quatre musiciens déguisés en fantômes d'un autre monde, on illustre la précarité de l'existence dans ce champ de bataille où l'être humain lutte pour trouver un sens à sa vie.

Avec délicatesse et vulnérabilité - sur scène, au studio de répétition, lors d'une discussion ou en tournée -, le Quatuor Bozzini construit pour la musique un avenir présent.

(Traduit par Vincent Collard) 